\title{
Necker cube: Duration of preexposure of an unambiguous form
}

\author{
PHILLIP L. EMERSON \\ Cleveland State University, Cleveland, Ohio 44115
}

\begin{abstract}
Two well practiced subjects indicated the apparent orientation of a Necker cube displayed immediately after the offset of one of two unambiguous forms. The unambiguous forms were identical to the Necker cube, except that lines were removed to represent opaque rather than transparent cubes. The duration of preexposure of the unambiguous forms was varied at $300,600,1,200,2,400$, and $4,800 \mathrm{msec}$. The main dependent variable was the estimated probability of the response to the ambiguous stimulus, being opposite to the orientation of the preexposed stimulus. From about 600 responses from each subject at each duration, these probability estimates turned out to be significantly different for the different durations. They all were greater than .5 and increased monotonically with preexposure duration. It was concluded that a simple random model of Necker reversals is inadequate; some form of a satiation model is needed.
\end{abstract}

A number of the past studies on reversals of the Necker cube, and similar phenomena, have been motivated by the desire to test the satiation theory of Köhler and Wallach (1944) and decide between it and/or various alternative explanations (see Cohen, 1959; Cornwell, 1976; Pelton, 1969; Sadler \& Mefferd, 1971). It is fair to report that the authors of these studies were not entirely in agreement concerning which theoretical conceptualization provides the best explanation for the experimental results. The reason for this might be that the phenomenon of spontaneous perceptual reversal is rather slippery to deal with experimentally. It is fraught with problems of biasing instructions to the subject, demand characteristics, motivational set, and wide variations among individuals with respect to prior practice. In addition, there is the question of what is to be measured. The most used dependent variable has been reversal rate, as measured by counting reported reversals over a period of seconds or minutes. In some studies (see Sadler \& Mefferd, 1971), the durations between reversals have been recorded. This free response procedure is somewhat lacking in controls, and the associated dependent variables would seem rather gross as indices of the underlying process. They have proven sensitive to effects of a number of experimental variables, such as stimulus size (Smith \& Barut, 1969) and complete vs. incomplete contours (Cornwell, 1976), as well as some individual differences variables (Wieland \& Mefferd, 1967). However, there seem to be more direct approaches to the testing of the satiation hypothesis and to the further experimental analysis of reversal phenomena.

The present study differs from many of the others in that spontaneous reversal rate was not measured. Indeed, the object was to reduce the spontaneity and to bring the momentary state of perception under experi- mental control. The general idea of the procedure was similar to that of Epstein and Rock (1960) and Leeper (1935), who investigated carry-over effects from prior exposures of unambiguous forms of the wife/mother-inlaw figure. The independent variable of main interest here was the duration of the prior exposure of one of two opposite unambiguous forms. The main dependent variable was the probability of giving the opposite response to the ambiguous form, as estimated from a run of 100 trials. Thus, satiation theory would predict these estimates to be generally greater than .5 and to increase with the duration of the prior exposure of the unambiguous form.

Sadler and Mefferd $(1970,1971)$ suggested that Necker reversals may be based on an overall stochastic rather than a satiation process. This idea can be sharpened into an extreme form, as follows. The simplest random process, untainted by any kind of satiation, would be one in which the probability of a reversal within a small time interval (rate parameter ${ }^{1}$ ) remains constant over time since the last reversal. Therefore, the hypothetical random process can be characterized by two parameters (for a given set of experimental conditions): (1) the rate constant for a reversal to state $y$, given that the present state is $\mathrm{x}$, and (2) the rate constant for a switch to $\mathrm{x}$, given that the present state is $y$. Thus, the past history of the process is irrelevant; elapsed duration of the present state has no effect on the imminence of a reversal. One implication is that the probability distributions for the durations of occurrences of states $\mathrm{x}$ and $\mathrm{y}$ both should be of the exponential form (McGill, 1967), but not necessarily of the same rate constant. Another implication is that experimental control of the state, and variation of its duration within an interval of time, should not affect the probability of either state immediately following the control interval. The latter implication 
is the general null hypothesis that was tested in the present experiments.

\section{METHOD}

\section{Apparatus and Display Geometry}

The experiments were conducted according to a program written for a PDP/9 computer with a microsecond real-time clock, a binary input buffer to detect responses from the subject, a binary output buffer to generate square-wave acoustical tones through a small speaker, and a Tektronix 502 CRT (without graticule) for the visual display.

The seated subject, with head position controlled by a chin and forehead rest, viewed the CRT at a horizontal distance of $110 \mathrm{~cm}$ from the vertical plane of the face of the CRT. However, the CRT was positioned $44 \mathrm{~cm}$ above eye level to reduce specular reflections of objects in the dimly illuminated room. The viewing distance was thus $118.5 \mathrm{~cm}$, and the line of sight formed an angle of $37 \mathrm{deg}$ from the normal to the plane at the center of the CRT.

The stimuli were obtained by the following operations. First, a 2 by 2 by 2 cube in standard position centered at the origin was defined by a list of the $(x, y, z)$ coordinates of its eight corners: $(1,1,1),(1,1,-1),(1,-1,1)$, and so on, where $x$ is the horizontal axis normal to the face of the CRT, $y$ is the horizontal axis within the plane of the face of the CRT, and $z$ is the vertical axis. The stimulus figures were orthogonal projections of the cube on the $\left(y^{\prime \prime}, z^{\prime \prime}\right)$ plane, after (1) numerical azimuth and elevation transformations (see Table 1) of the corners of the cube, (2) multiplication by a size factor, (3) addition of location constants for centering on the CRT, and (4) linear interpolation of many points between appropriate corners to obtain the apparently continuous edges of the cube. The same azimuth and elevation were used throughout (i.e., 25 and $10 \mathrm{deg}$, respectively). For the ambiguous form, all 12 edges of the cube were displayed. There were two unambiguous forms, and they were labeled "top view" and "bottom view" in the instructions to the subjects. They were identical to the ambiguous form, except that in each case an appropriate set of three edges was omitted to represent views of an opaque cube. The shapes are illustrated in Figure 1. The stimulus size was made rather small to reduce possible effects of the subject's developing habits of fixating on particular parts of the figure. The vertical edges measured $1.5 \mathrm{~cm}$ on the face of the CRT. By means of a real-time clock, the scan rate was made the same for ambiguous and unambiguous stimuli, even though different numbers of points had to be plotted in the two cases. It was fast enough $(66.67 \mathrm{~Hz})$ that there was no apparent flicker.

\section{Procedure}

Two experiments were conducted using the same subjects and essentially the same procedure. Each run of trials took about $30 \mathrm{~min}$ and consisted of 100 trials, on the basis of which probability estimates were calculated. In the first experiment, each trial consisted of six stages: (1) presentation of a randomly chosen member of the pair of unambiguous stimuli (with equal

Table 1

Equations Used to Rotate the Necker Cube with Respect to Azimuth $(\theta)$ and Elevation $(\Phi)$

\section{Azimuth}

$\mathrm{x}^{\prime}=\mathrm{x} \cos \theta+\mathrm{y} \sin \theta$

$y^{\prime}=y \cos \theta-x \sin \theta$

$\mathrm{z}^{\prime}=\mathrm{z}$

Elevation

$\mathrm{x}^{\prime \prime}=\mathrm{x}^{\prime} \cos \Phi+\mathrm{z}^{\prime} \sin \Phi$

$y^{\prime \prime}=y^{\prime}$

$\mathrm{z}^{\prime \prime}=\mathrm{z}^{\prime} \cos \Phi-\mathrm{x}^{\prime} \sin \Phi$

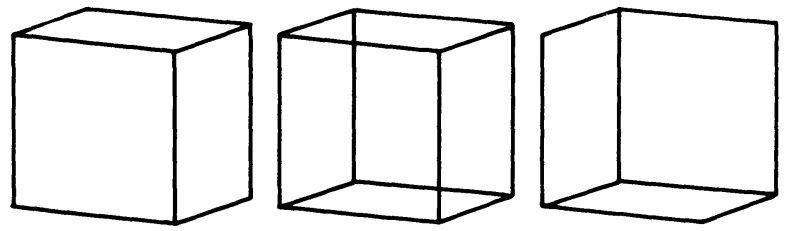

Figure 1. Shapes of the stimuli. From left to right: top view, ambiguous, bottom view.

probabilities) for one of five preselected durations, (2) intersignal interval (ISI) of $50 \mathrm{msec}$, (3) presentation of either the ambiguous stimulus (probability $=.5$ ) or one of the unambiguous stimuli (probability $=.25$ for each) for $480 \mathrm{msec}$, (4) response stage, in which the subject responded with a triplethrow spring-loaded center-rest switch (up for "top view" and down for "bottom"), (5) 250-msec interval for a 500-Hz clearly audible error tone, which was sounded if the second stimulus was unambiguous and the response did not match it, and (6) intertrial interval of $5 \mathrm{sec}$ minus the duration of the first stimulus.

The 50-msec ISI of the second stage was a compromise. If it were too long, carry-over effects would be lost; if it were too short, there would be failure to detect the offset of the first and the onset of the second stimulus. The random intermixture of unambiguous stimuli (training trials) and ambiguous stimuli (test trials) in the third stage decreased the rate of data collection but ensured that the proper response coding was maintained, since the error tone of the fifth stage signaled any lapses in adherence. The intertrial interval (six th stage) was made equal to $5 \mathrm{sec}$ minus the duration of the first stage in order to avoid confounding the effects of preexposure duration with the total duration of a 100-trial run. This was necessary because the preexposure duration was held constant within each run.

The second experiment differed from the first only in that the third stage of each trial was terminated by the response, rather than having a fixed duration of $480 \mathrm{msec}$. Each experiment consisted of six blocks of five runs each, for each of two subjects. Within each block, a run was made with each of the five preexposure durations: $300,600,1,200,2,400$, and $4,800 \mathrm{msec}$. The order of testing with these five durations was randomized independently for each block-subject-experiment combination. The subjects were two normal adult males who had had much prior practice, having participated in a pilot experiment using the same stimuli and apparatus and a very similar procedure. The subjects were instructed to observe both stimuli on each trial and to respond by moving the switch up if the second stimulus appeared in its top-view aspect or down if it appeared in its bottom-view aspect. They were instructed to choose arbitrarily if the second stimulus appeared in neither or both aspects. They were initially instructed to respond as rapidly as possible and that their reaction times would be measured. However, there was no reaction time feedback during the course of the experiments.

\section{RESULTS}

For the main analysis, only the data from test trials were used. An "opposite" response was defined as a bottom-view response to the ambiguous stimulus following a top-view unambiguous stimulus or a top-view response to the ambiguous stimulus following a bottomview unambiguous stimulus. For each run of 100 trials, the probability of "opposite" was estimated as the number of "opposite" responses divided by the number of test trials. These probability estimates were averaged within each duration-experiment-subject combination, 
and an analysis of variance was performed on the averages. The test of the durations main effect yielded significance $[F(4,4)=52.29]$, but the experiments effect and the Durations by Experiments interaction did not. The means of the probability estimates in increasing order of the preexposure durations were $.57, .64, .73, .78$, and .85. Analysis of the data for each experiment separately yielded significance of the durations effect in each case. In both cases, also, the trend of the means was monotonic increasing, not markedly different from that of the combined data.

The results of some secondary analyses are as follows. There was an overall bias for the top-view response to the ambiguous figure. An analysis of variance of the estimated probabilities of a top-view response yielded significance only for the preexposure durations main effect $[F(4,4)=61.04]$. The mean estimated probabilities in increasing order of the five durations were $.74, .75, .70, .67$, and .64 . In the second experiment, reaction times were measured on every trial, and from each run of 100 trials, mean reaction times were calculated for (1) training trials and (2) test trials. These means were averaged over runs with corresponding preexposure durations and subjected to analysis of variance in a 2 (trial types) by 5 (durations) by 2 (subjects) design. Only the main effect for trial types was significant $[F(1,1)=184]$. The mean reaction times for the two trial types were $571 \mathrm{msec}$ for training trials and $1,058 \mathrm{msec}$ for test trials.

\section{DISCUSSION}

The monotonic increase of the probability of an "opposite" response, as a function of the duration of the preexposed unambiguous stimulus, provides strong grounds for rejecting the purely random model as a description of Necker reversals. Even if the random model were generalized to allow the time constants to vary gradually over time, perhaps as a function of practice, it could not account for the present results. Whether realized as satiating electrical currents in the brain or otherwise, it seems clear that there is a short-term growth of reactivity during the experience of one of two possible perceptual states in the presence of the Necker cube. To modify the random model accordingly would require allowing the rate constants to vary as a function of time since entry into the current perceptual state. ${ }^{2}$ But that turns it into a satiation model.

It is not surprising that the probability of top-view responses also varied with preexposure duration. This result is entirely to be expected in view of the experimental design and the effects on the probability of "opposite" response. Most subjects and casual visitors have reported that the "top view" of this version of the Necker cube is easier to see than is the "bottom." Thus, there is a fundamental bias that decreases when the duration of the preexposure is increased, simply because the probabilities of the two unambiguous stimuli are equal. If perfect experimental control of the momentary state of perception were achieved, perhaps by further extending the preexposure duration, the probability of top-view responses would have to asymptote at .5 , under the present experimental procedure. ${ }^{3}$

It was thought that reaction times might also reflect some effects of the durations. That they did not may be due to the fact that there was no feedback of reaction-time information during the long course of the experiments. The mean of $571 \mathrm{msec}$ for the training trials is rather long for an easy discrimination such as this, and it is suspected that the subjects were attending more to accuracy than to speed.

Although the above interpretations of the results seem well justified, there is one puzzle that they do not address. In dabbling with differential equations to describe the dynamics of Necker reversals, the author has come to the tentative conclusion that if there is a negative reactive force, as implied by the present results, then there must also be a positive cohesive one to temporarily hold the current Gestalt together. It would be cancelled only after the reactivity had grown sufficiently over time since the last reversal. Clearly, there is a temporary lock-in phase in the behavior of Attneave's (1976) multivibrator model. Moreover, the present results are exactly the opposite of those of Epstein and Rock (1960) and Leeper (1935). Using the wife/ mother-in-law figure, they found evidence only for the cohesive tendency. The responses to the ambiguous form were almost always the same as those to the preexposed unambiguous form. A conjecture worth exploring experimentally is as follows. In the present experiments, the trials were well randomized, so that if there were trial-to-trial carry-over effects, the carry-over would be as likely in one direction as in the other. Unfortunately, this does not ensure against biasing effects of the carry-over. On half the trials (those trials in which the carry-over is opposite to the unambiguous stimulus), the duration of the preexposure perceptual state would be just as long as that of the stimulus preexposure. But on the other half, it would be effectively longer because the carry-over would be in the same direction as the preexposed stimulus. This bias would work to increase the frequency of "opposite" responses for short preexposure durations and to conceal the cohesive effects, if they exist.

The wife/mother-in-law figure is much more complex than the Necker cube; it contains representations of human faces, and the third perceptual dimension does not seem to be involved. Also, in the procedures of Epstein and Rock (1960) and Leeper (1935), there were only a dozen or so exposures to the subject. In the present experiments, the subjects had experienced at least 1,400 exposures of the ambiguous stimulus prior to data collection. In the present experiments, too, the stimulus was made smaller than has been usual (about 1 deg of arc), because in informal viewing it had seemed possible to achieve much voluntary control of the perceptual state with large figures, by fixating on particular parts of the figure. It seems likely that some of these differences promoted the cohesive component in those experiments, but the reactive one in the present experiments.

\section{REFERENCES}

Attneave, F. Multistability in perception. In R. Held \& W. Richards (Eds.), Recent progress in perception: Readings from Scientific American. San Francisco: Freeman, 1976.

CoHen, L. Rate of apparent change of a Necker cube as a function of prior stimulation. American Journal of Psychology, 1959, 72, 327-344.

CoRnwell, H. G. Necker cube reversal: Sensory or psychological satiation? Perceptual and Motor Skills, 1976, 43, 3-10.

Epstein, W., \& Rock, I. Perceptual set as an artifact of recency. American Journal of Psychology, 1960, 73, 214-288.

Köhle R, W., \& Wallach, H. Figural aftereffects of visual processes. Proceedings of the American Philosophical Society, 1944, 88, 269-357.

LEEPER, R. A study of a neglected portion of the field of learningThe development of sensory organization. Journal of Genetic Psychology, 1935, 46, 41-75. 
McGILL, W. J. Stochastic latency mechanisms. In R. D. Luce, R. R. Bush, \& E. Galanter (Eds.); Handbook of mathematical psychology (Vol. 1): New York: Wiley, 1967.

Pelton, L. H. Reversals in the momentary absence of percepts. Perceptual and Motor Skills, 1969, 29, 677-678.

Sadler, T. G., \& Mefferd, R. B., JR. Fluctuations of perceptual organization and orientation: Stochastic (random) or steadystate (satiation)? Perceptual and Motor Skills, 1970, 31, 739-749.

Sadler, T. G., \& MefFe RD, R. B., JR. Data requirements for satiation theories: A rejoinder to Price. Perceptual and Motor Skills, 1971, 33, 999-1005.

SмIтн, O. W., \& BARUT, C. Relation of spatial abilities to reversal rates of Necker cubes varying in degree of completeness. Perceptual and Motor Skills, 1969, 29, 760-762.

Wieland, B. A., \& MefFerd, R. B., JR. Individual differences in Necker cube reversal rates and perspective dominance. Perceptual and Motor Skills, 1967, 24, 923-930.

\section{NOTES}

1. More properly, the limit of the ratio of the probability to the small time interval, as the small time interval approaches zero. Unlike probabilities, such rate constants can exceed unity.

2. For example, suppose the rate parameters to grow from zero in proportion to time since the last reversal. Let the (cumulative) probability distribution function for the duration of one of the states be denoted by $F(t)$ and its derivative (the frequency function) by $f(t)$. Then $f(t) /[1-F(t)]=g t$ (see McGill, 1967), where $g$ is the growth factor. The solution to this equation is $\dot{F}(t)=1-\exp \left(-g t^{2} / 2\right)$, for $0 \leqslant t<\infty$. The density, $f(t)=g \operatorname{texp}\left(-\mathrm{gt}^{2} / 2\right)$, is nearly linear with slope $g$, for small $t$. The mode and median are $1 / \sqrt{\mathrm{g}}$ and $1.1774 / \sqrt{\mathrm{g}}$, respectively. The mean and standard deviation are $1.2533 / \sqrt{\mathrm{g}}$ and $.655 \times 1 / \sqrt{\mathrm{g}}$, and the moment-generating function is

$$
\mathrm{M}(\theta)=\sqrt{2 \pi / \mathrm{g}} \theta \exp \left[\theta^{2} /(2 \mathrm{~g})\right] \Phi(-\theta / \sqrt{\mathrm{g}})+1,
$$

where $\Phi(\mathrm{z})$ is the normal distribution function. The distributions for the durations of the two different states would be of the same form, but most likely with different values of $\mathrm{g}$.

3. The fundamental top-view bias could not have caused the observed effects of durations on the probability of "opposite" responses. Nor would a simple decrease of top-view responses necessarily increase the frequency of "opposite" responses. The probability of "opposite" responses starts at .5 (a priori) with zero preexposure duration and increases, whereas the probability of top-view responses starts at around .75 and decreases. Any effect of one upon the other would have to be in moving the probability of the other toward $1 / 2$.

(Received for publication October 22, 1979.) 